\title{
Geometric Clustering for Line Drawing Simplification
}
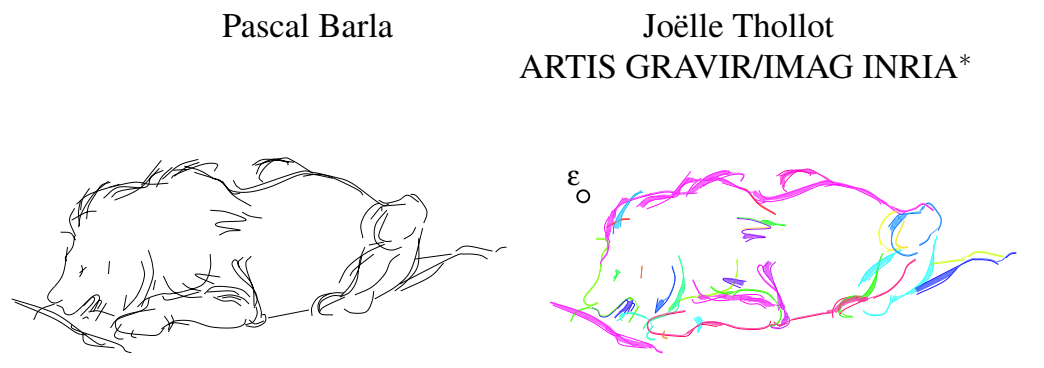

\author{
François X. Sillion
}

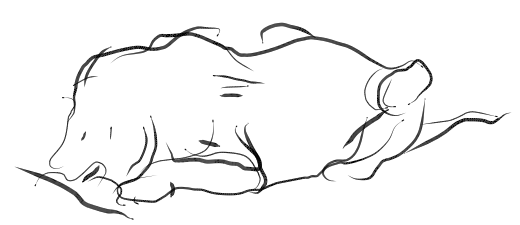

Figure 1: Lines of the initial drawing (left) are first automatically clustered into groups that can be merged at a scale $\varepsilon$ (middle). A new line is then generated for each group in an application-dependent style (at right, line thickness indicates the mean thickness of the cluster).

\section{Introduction}

We present a new approach to the simplification of line drawings, that maintains the morphological structure of the original drawing while decreasing the number of lines. The technique works by analyzing the structure of the drawing at a certain scale and identifying clusters of lines. These clusters are then processed to create new lines in a separate stage where different scenarios can be favored based on the target application.

This two-stages decomposition (see Figure 1) naturally adapts to various applications: density reduction of a drawing, where too many lines project in a given region of the image; Level-ofdetail (LOD) representations for line-based rendering (contours and hatching), where the number of lines must vary with scale; and progressive editing, where the user refines a curve by successive sketches, viewed as an iterative simplification of the set of line sketches drawn by the user. Our goal is to provide a low-level tool common to these applications.

Two research fields have addressed this problem: density reduction of a 3d line-drawing (e.g. [Grabli et al. 2004]) where, in general, the simplification process is restricted to the deletion of the least significant lines; and perceptual grouping (e.g. [Rosin 1994]) studying the way a human naturally groups the lines in an image. Our method combines some principles of perceptual grouping (proximity, continuation and parallelism of lines) with the density reduction approaches found in computer graphics. Our main contribution is a generic clustering algorithm which serves as a basis for the envisioned applications.

\section{Exposition}

We have identified two common properties of the simplification methods that preserve the shape of the original drawing. First the overall configuration of the original drawing needs to be respected in the simplified one. This so-called coverage property consists of creating new lines only in regions where initial lines can be found. Second, the new lines have to respect the way the initial lines have been created. This is similar to imposing a morphological property that prevents new lines from folding onto themselves. These two properties correspond to the proximity, continuation and parallelism criteria found in perceptual grouping.

We consider a drawing to be a digital image composed of a number of vectorized $2 \mathrm{~d}$ lines. Such images can be obtained in various ways (scanned, digital input, contour extraction, NPR) and lines can hold any type of attribute.

*ARTIS is a team of the GRAVIR/IMAG research lab (UMR C5527 between CNRS, INPG, INRIA and UJF), and a project of INRIA.
Our clustering algorithm iteratively groups pairs of lines that satisfy the coverage and morphological properties. A simplification scale $\varepsilon$ is chosen (small circle on the figures). Then, following our morphological property, we define an $\varepsilon$-line as a line that does not fold onto itself at the scale $\varepsilon$. From our coverage property, we define an $\varepsilon$-group as a group of lines that can be covered by a single $\varepsilon$-line, based on the symmetric Hausdorff distance. A pair of lines will then be clustered if and only if it is an $\varepsilon$-group. Our clustering also takes into account the attributes of the original lines (thickness, color, ...) by means of an error measure.

Finally, the abstraction stage creates a single line for each cluster. A specific strategy for the line creation can be defined to adapt to the target application.

We demonstrate our results on three applications: progressive drawing, density reduction (see Figure 1) and level-of-detail (see Figure 2). Two simple abstraction strategies are used: creating an "average" line or selecting one of the lines in the cluster as a representative. The clustering stage takes a few seconds, and increases with the number of input lines and the scale parameter $\varepsilon$.

These results demonstrate both the genericity of the clustering stage and adaptability of the abstraction stage.

\section{References}

Grabli, S., Durand, F., And Sillion, F. 2004. Density measure for line-drawing simplification. In Proc. of Pacific Graphics.

Rosin, P. 1994. Grouping curved lines. In 5th British Machine Vision Conf, pp. 265-274.
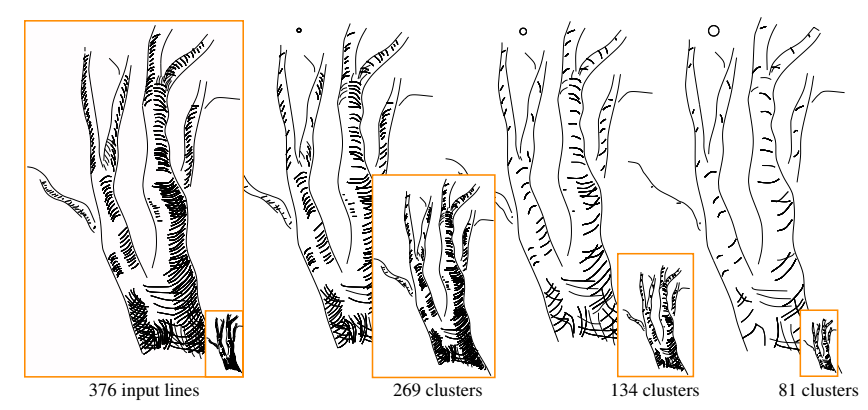

Figure 2: A series of LODs made by progressively increasing $\varepsilon$ and clustering the previous level. We use two different strategies: we keep the longest line of each cluster for the hatchings and create an average line for the contours. 